\title{
Classification of skin cancer images by applying simple evolving connectionist system
}

\author{
Al-Khowarizmi', ${ }^{1}$ Suherman ${ }^{2}$ \\ ${ }^{1}$ Department of Information Technology, Universitas Muhammadiyah Sumatera Utara, Indonesia \\ ${ }^{2}$ Department of Electrical Engineering, Universitas Sumatera Utara, Indonesia
}

\begin{tabular}{|c|c|}
\hline Article Info & ABSTRACT \\
\hline Article history: & \multirow{9}{*}{$\begin{array}{l}\text { Simple evolving connectionist system (SECoS) is one of data mining } \\
\text { classification techniques that recognizing data based on the tested and the } \\
\text { training data binding. Data recognition is achieved by aligning testing data to } \\
\text { trained data pattern. SECoS uses a feedforward neural network but its hidden } \\
\text { layer evolves so that each input layer does not perform epoch. SECoS } \\
\text { distance has been modified with the normalized Euclidean distance formula } \\
\text { to reduce error in training. This paper recognizes skin cancer by classifying } \\
\text { benign malignant skin moles images using SECoS based on parameter } \\
\text { combinations. The skin cancer classification has learning rate } 1 \text { of } 0.3 \text {, } \\
\text { learning rate } 2 \text { of } 0.3 \text {, sensitivity threshold of } 0.5 \text {, error threshold of } 0.1 \text { and } \\
\text { MAPE is } 0.5184845 \text { with developing hidden node of } 23 \text {. Skin cancer } \\
\text { recognition by applying modified SECoS algorithm is proven more } \\
\text { acceptable. Compared to other methods, SECoS is more robust to error } \\
\text { variations. }\end{array}$} \\
\hline Received Sep 13, 2020 & \\
\hline Revised Mar 21, 2021 & \\
\hline Accepted Apr 9, 2021 & \\
\hline Keywords: & \\
\hline Classification & \\
\hline Data mining & \\
\hline SECoS & \\
\hline Skin care & \\
\hline
\end{tabular}

This is an open access article under the CC BY-SA license.

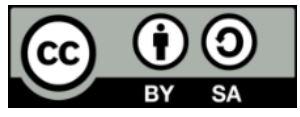

\section{Corresponding Author:}

Suherman

Department of Electrical Enfineering

Universitas Sumatera Utara

Jalan Almamater Kampus USU Medan, Indonesia

Email: suherman@usu.ac.id

\section{INTRODUCTION}

Classification is one of the data mining algorithms that aims to enable computer or processor to carry out learning by methods and algorithms to fit into a machine learning concept [1]. Classification involves training and testing data [2] in form of text, images, and videos [3]-[5]. Learning process in defining the model may involve data properties that referred to as data features, so that learning process exerts identification, decision support, and method improvement [6]. Classification has become one of the major focuses on computer science. Data mining algorithm is able to analyze complex dataset and to predict various newly inputted data by not making general assumptions on data distribution. In support to data mining study, complex data in various applications have been collected for classification study purposes that are referred to as datasets [7]. Dataset may in form of text records, literature images, old scriptures, speech records, business data, pharmaceutical data or medical images.

Skin cancer is the primary cancer desease in some countries with death rate is up to $10 \%$ [8]. Number of skin cancer patients increases steadily every year. Skin disease detection and examination are important to determint the correct treatment. However, classification study on cancer images still requires accuracy prediction that has been research challenges, including research on skin disease classification algorithm. However, low level feature extractions for skin deseases based on shape, texture, and color properties extracted from the lesion image are still considered as lack of clinical meaning [9]. High level 
clinical oriented features are expected to contribute more by applying various color normalization approaches. Techniques such as content-based image retrieval [10] and short term of lesions monitoring are expected to provide clinical meaningful dataset and to enrich the classification diagnostic. Moreover, if the rich visual feature is complemented by the clinical metadata, diagnostic accuracy may increase and treatment may be proper.

Traditional classifier such as support vector machine (SVM) is widely used to classify skin cancer images. For instance, Alquran et al. [9] skin disease analysis using SVM with principal component analysis (PCA) to classify skin disease dataset. Feature extractions were performed in four steps (RGB image, binary image, lesion image and histogram equalized image) to get kurtosis, contrast, mean, skewness, energy, circulation, standard deviation, homogeneity and correlation.. The features were then fed into PCA, before SVM classification was performed. The five selected feature exterted the accuracy of $92.1 \%$. Meanwhile, Chung et al. [11] used SVM with the help of persistence diagrams from persistent homology algorithm (which is part of the topological data analysis (TDA)) for segmenting images. The method also combines the persistent homology with the persistence stats with the Betti and entropy curves before classified by using SVM. Best validation score was achieved when XYZ curves, XYZ stats, RGB stats were used as selected features.

However, traditional classifiers such as SVM, neural network and decision tree may have difficulties in dealing with large dataset and frequent variation in fault pattern. Although these traditional classifier may result high accuracy for given dataset, future complex and rich dataset require more efficient classifier. Simple evolving connectionist system (SECoS) or so called as evolved multilayer perceptron [12] is the smallest realization of ECoS that has capability in forecasting big data pattern. This method is powerfull in processing the rich dataset. SECoS applies the ECoS principle by developing the multilayer preceptron (MLP) method [13]. SECoS is sometimes referred to as the evolving multilayer perceptron (eMLP) [14]. SECoS has three layers, namely input, hidden and output layerr [15]. The input layer processes raw input data for testing and training. The hidden layer calculates the SECoS method by using some formulas that have been developed in accordance to the ECoS principle. The process is carried out evolvely where the hidden layer increases the object properties to get the specified value. The output layer is to get classification value [16]-[18].

SECos is able to perform data classification in various fields [19]. The work in [20] used SECoS to classify stroke data texts to help patient treatment by determining how much fibrinogen should be consumed. The classification process is very helpful in assisting doctors and patients. In addition, the method has also been used to classify images of the Qur'an verses to help readers understanding the tajweed law in reading the Qur'an [21]. Since data is not a text type, feature extractions were performed [22], [23]. SECoS can be integrated into a single image processing module [24], [25], for instance, to isolate phoneme recognition [26] where large data patten can be memorized and new inputted data can be adapted. SECoS reduces a large number of hidden nodes. SECoS has also been used for adaptive speech recognition, business analysis, as well as health and pharmaceutical analysis [27]. This paper identifies skin cancer from a balanced skin cancer images dataset of malignant and benign skin images.

\section{MATERIAL AND METHOD}

\subsection{Dataset}

The employed data set in this paper is based on previous research [28] that taken from a balanced skin cancer image dataset of malignant and benign skin images. This skin cancer dataset is accessible and available online at kaggle. The images were gathered by the collaboration of skin images (ISIC) for study and implementation purposes. There are 360 images: $16.7 \%$ falls under the category of malignant melanomas and $83.3 \%$ is in benign category.

The classification is performed based on the $224 \times 224$ colored images. The dataset itself contains 1800 datasets, where 136 datasets with 100 training data and 36 testing data were selected. The classification targets are "benign" and "malignant". In order to fasten algorithm execution time, the $224 \times 224$ colored image was converted into $28 \times 28$ grayscale image.

\subsection{SECoS architecture}

Figure 1 shows the common architecture of SECoS algorithm in classifying skin cancer discussed throughout this paper. The architecture describes steps involved in classification such as grayscaling, feature extraction, data training and data testing.

Initially, data set is splitted into training and testing data where the distribution of bboth benign and malignant data are provided. These images are then converted into grayscale images. A grayscale image is represented by a single sample value in each pixel to represent the amount of light. Grayscale image can be in 
form of black/white or gray monochrome image. Black is the weakest light intensity and white is the strongest. Next is to do feature extraction with the oriented gradient histograms (HOG). HOG feature extraction determines the frequency of gradient orientation in a portion of an analyzed image by using contrast normalization. This gradient field captures the image spatial structure. HOG descriptors are familiar to be used on computer vision and recognition. HOG descriptors extract features for machine learning algorithm. HOG feature extraction extracts grayscale images features to be used by SECoS algorithm.

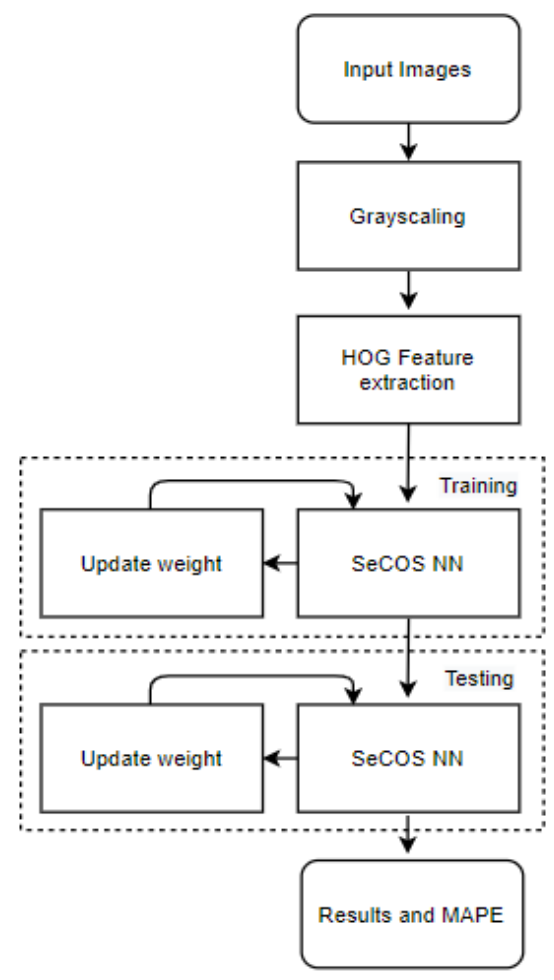

Figure 1. General architecture

Data is then trained in SECoS and the weight is updated. If the weight is out of scope, then it returns to SECoS, but if it is correct then next step is performed. Data testing in SECoS is performed by updating weights. If the weight is correct, the next step is performed. MAPE is calculated by using (1), where actual value, classification value and number of data are represented by $a, b$, and $n$ subsequently.

$$
M A P E=\frac{\sum_{t=1}^{n}\left|\frac{a-b}{a}\right|}{n} \times 100 \%
$$

\subsection{SECoS development on normalized euclidean distance}

SECoS has a neural network that extends to the hidden layer and the output layer as described in Figure 2(a). Figure 2(a) shows that three three layers of neuron processes. The first layer is the input layer, followed by the evolving or constructive stage as the second layer and output layer as the fial stage. The first layer correlates to all second layer. The second layer adds neurons and proceeds learning. The evolved layer neurons are turned on according to distance of Eucledian between the input part of SECoS and the evolving layer. Finally, the results go to output layer. SECoS is fully connected network where the connections number from input to evolving layer equals to the number of input neurons. By using the tested patient data of $-455 \mathrm{G} / \mathrm{A}$ polimorphism of beta fibrinogen gene, the normalized Euclidean has the smallest MAPE compared to Hamming and Manhattan distances as shown in Figure 2(b). The performance differences show that the normalized Euclidean distance has the smallest MAPE. Although difference is lower than 1.8\% which means that distance formula exerts insignificant effect on the training results accuracy, Euclidean will be employed in this reasearch. Therefore the Euclidean distance can be directly calculated. Since distance in the range of $[0,1]$, input data should be normalized [29]. 


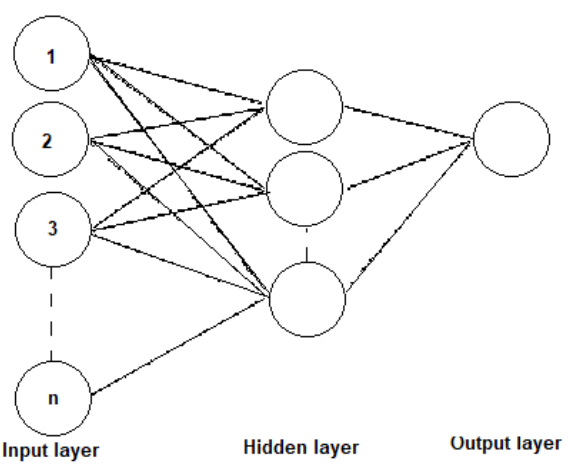

(a) SECoS layers

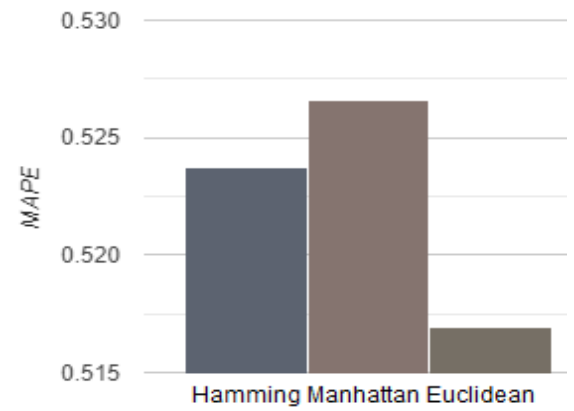

(b) Normalized distance performance comparison

Figure 2. SECoS neural network and distance comparison [30]

In general, feedforward networks have an activation function. But, SECoS uses an activation function that is linear and called as activation $\mathrm{A}$ at the evolving layer node $\mathrm{n}$ that is determined by (2).

$$
A_{n}=1-D_{n}
$$

An is an activation function at nodes $\mathrm{n}$ and $\mathrm{Dn}$ which is determined by using the normalized calculated distance that lies in between of input weight and the output weight at the destination node. Generally, Dn is a normalized Hamming distance, but this paper refers to [31] which has carried out an optimization by changing the normalized Hamming distance to normalized Euclidean distance which has better results in learning. The normalized Euclidean distance formula is given by (3), where K, nodes number at the time of SECoS network input, I is the value that is inputted by incoming vector, $\mathrm{W}$ is the matrix of the evolving layer weight.

$$
\mathrm{D}_{\mathrm{n}}=\sqrt{\sum_{\mathrm{i}}^{\mathrm{K}} \frac{\left|\mathrm{I}_{\mathrm{i}}-\mathrm{W}_{\mathrm{i}}\right|^{2}}{\mathrm{k}}}
$$

Further, learning was performed by analyzing images on the skin cancer dataset. The SeCOS learning process injects neurons to the evolved layer only if the value of the linear activation is smaller than the threshold of sensitivity or if the calculated error is much higher than the threshold. The outgoing and incoming connection weight of the introduced neuron is related to the vectors input and output. Otherwise, the weight of connection is adjusted to ensure that the analyzed neuron approximates the input vector. The journey of SECoS is outlined by [30]. The vector of input I is fed into the feedforward matrix, if the activation value reaches maximum of $\mathrm{A}_{\max }$ in the node has value smaller than the threshold of sensitivity $\left(S_{\text {thr }}\right)$, then a new node will be added to the covered layer. Otherwise, SECoS calculates the difference value of the input and output vectors, $\mathrm{O}_{\mathrm{c}}$ and $\mathrm{O}_{\mathrm{d}}$. If the different is higher than the threshold error coefficient $\left(\mathrm{E}_{\text {trh }}\right)$ or if the expected output node is unavailable, then SECoS adds a new node to the hidden layer. Otherwise, winning node on hidden layer should update its connection weight. The aformentioned step is performed for each input without an epoch.

A node should be added only if the connection weight has a value following the input vector I. Output vector weigth is set folowing to the output vector Od. The process of w feedforward from the covered layer to the output is performed in two different ways. The first way is by using the propagation method of One-of-N. This propagation is executed only by the covered node with has the maximum value. The adjustment of the winning node entry weight $\mathrm{j}$ is performed by using (4).

$$
W W_{i i,}(t t+1)=W W_{i i,}(t t)+\eta \eta_{1} I I_{i i}-W W_{i i, j}(t t)
$$

The inputted weight at $i i$ is denoted as $W W_{i i, j j}(t t) j j$ and $W W_{i i, j j}(t t+1)$ is the weight at $(t t+1)$. Meanwhile, $\eta \eta_{1}$ is the learning rate 1 value and $I I_{i i}$ is the $i$ value of input vector $\mathrm{I}$. The adjustment of the output weight in node $\mathrm{j}$ is performed based on (5).

$$
(t t+1)=W W j j, p p(t t)+\eta \eta 2 A A j j \times E E p p
$$


Where $W W_{j j, p p}(t t)$ is the value of the exit weight at $j j, p p$ when $(t t), W W_{j j, p p}(t t+1)$ is the value of the weight entered at $j j, p p$ when $(t t+1), \eta \eta_{2}$ is the learning rate $2, A A_{j j}$ is the activation value of the node.

\section{RESULTS AND DISCUSSIONS}

At this stage, the skin cancer classification process for patients with malignant and benign moles has been carried out using the modified SECoS method. The initial process includes dataset collection in form of images of malignant and benign skin moles. Figure 3 shows the process of grayscale image convertion and HOG feature extraction of malignant and benign skin moles before fed into SECoS classifier. The grayscale of dataset images is adjusted into the desired color, such as monochromatic colors from black to white. By doing this, the grayscale image only has gray and is colorless. The feature extractions were then performed by using the HOG feature extractor with the help of tools created with python syntax.

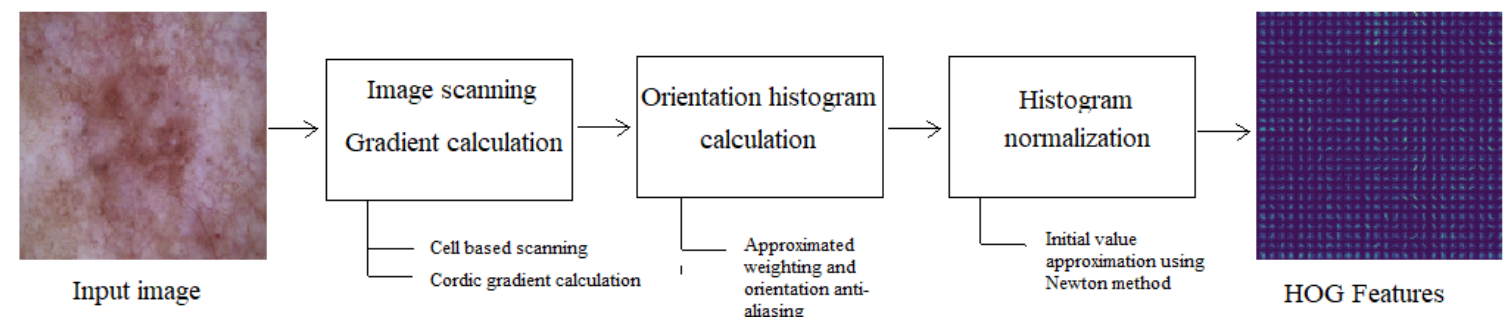

Figure 3. HOG feature extraction

Figure 4 shows sampel of malignant skin moled and benign skin moles and the HOG of those images. Figure 4(a) and 4(c) show examples of malignant and benign skin mole images used in the experiment. These images describe brushes caused by skin cancer. These images are then converted into HOG images as shown in Figure 4(b) and 4(d), which pose more uniform patterns. The HOG images as SECoS input have normalized values in range of 0 to 1 . In testing stage, the process is similar to the calculation in SECoS. First, calculate all parameter values for both learning rates, threshold of sensitivity and error and the lot of the training data. Then the propagation is performed starts from input to hidden layers by determining the values of activating function including the normalized Euclidean value. The propagation starts from hidden to the output layer is performed afterward. The calculated MAPE value is determined from the training data based on the pattern that has been obtained. Both training and testing processes performed in this paper use 136 randomized images data on malignant and benign moles images. About 100 testing data and 36 training data are depicted in Figure 5.

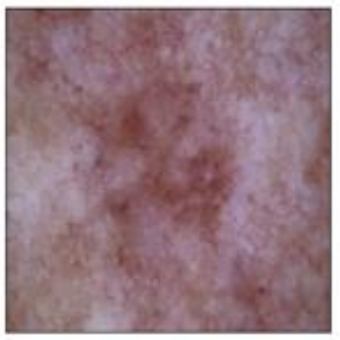

(a)

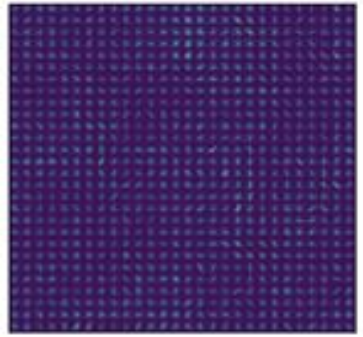

(b)

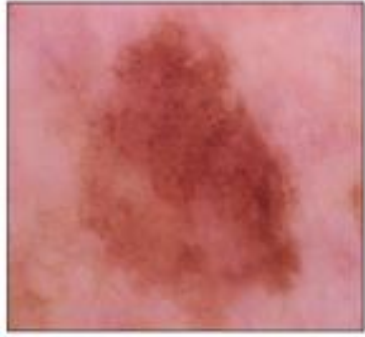

(c)

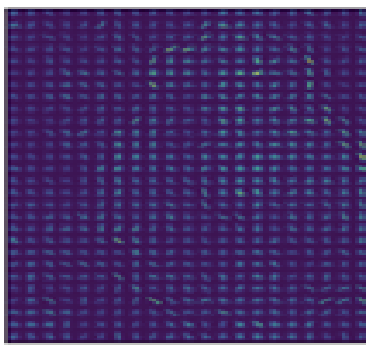

(d)

Figure 4. Sample of skin cancer images and their HOGs, (a) malignant skin moles, (b) HOG of malignant skin moles, (c) benign skin moles, (d) HOG of benign skin moles

Figure 5 shows training process. There is a dataset on the upper left which is benign and malignant skin images data, the lower left dataset is normalized data for benign and malignant skin moles with a range of 0 to 1 and the dataset list on the right is the result of propagation from hidden layer to the output layer. The output layer also results the calculated MAPE value from the amount of data being trained. Figure 6 shows 
the virtual results of the testing data. The generated MAPE produced the minimum results when input parameter value of learning rate 1 is 0.3 , learning rate 2 is 0.3 , the sensitivity threshold is 0.5 and the error threshold is 0.1, producing MAPE of 0.5184845 as shown in Figure 6(a). Meanwhile, the number of hidden nodes is 23 for given minimum MAPE as depicted in Figure 6(b).

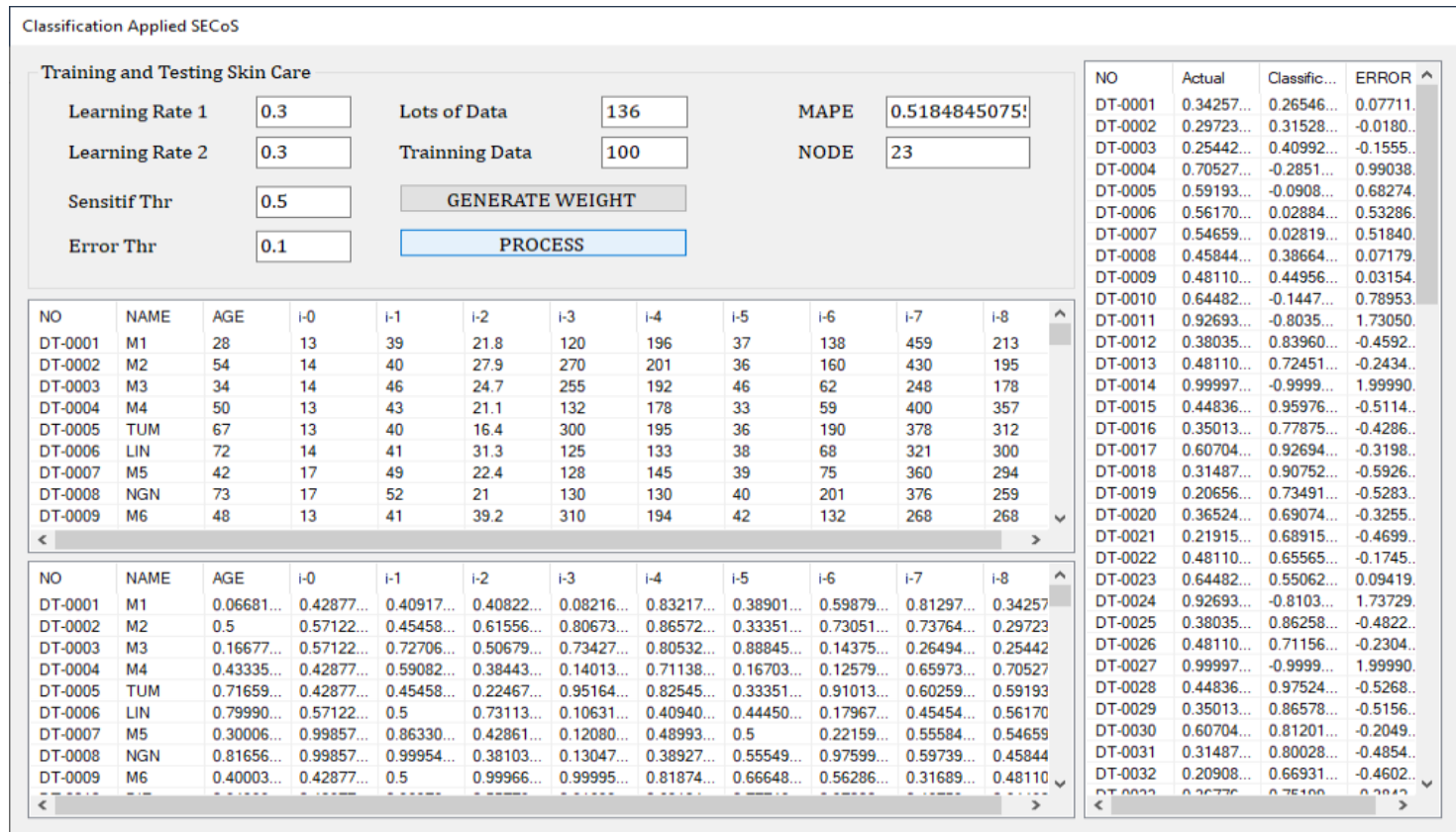

Figure 5. Skin cancer pattern training

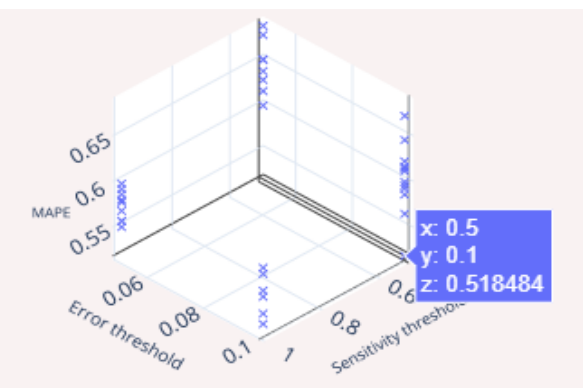

(a)

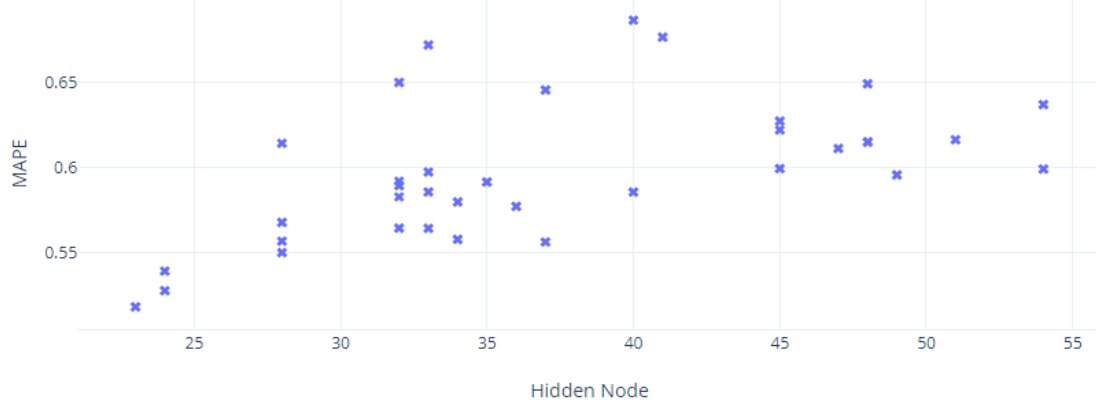

(b)

Figure 6. Visual results of skin cancer testing, (a) MAPE vs error threshold and sensitivity threshold, (b) MAPE vs Number of hidden node

Int J Artif Intell, Vol. 10, No. 2, June 2021: $421-429$ 
Table 1 shows the combination of parameters in the modified SECoS method where the activation function involves the normalized Euclidean distance formula. The learning rate varies from 0.3 to 0.9 , the sensitivity threshold is from 0.5 to 0.99 and error threshold is from 0.05 to 0.1 . The number of hidden nodes is changing dynamically as SECoS algorithm continuously learns from the input received and performs adaptive learning.

Table 1. Classification result with modified SECoS

\begin{tabular}{|c|c|c|c|c|c|}
\hline Learning Rate 1 & Learning Rate 2 & Sens. Threshold & Error Threshold & MAPE & Node \\
\hline 0.3 & 0.3 & 0.5 & 0.1 & 0.614285 & 28 \\
\hline 0.3 & 0.6 & 0.5 & 0.1 & 0.671923 & 33 \\
\hline 0.3 & 0.9 & 0.5 & 0.1 & 0.645498 & 37 \\
\hline 0.6 & 0.3 & 0.5 & 0.1 & 0.585737 & 40 \\
\hline 0.6 & 0.6 & 0.5 & 0.1 & 0.622073 & 45 \\
\hline 0.6 & 0.9 & 0.5 & 0.1 & 0.611276 & 47 \\
\hline 0.9 & 0.3 & 0.5 & 0.1 & 0.599489 & 45 \\
\hline 0.9 & 0.6 & 0.5 & 0.1 & 0.595727 & 49 \\
\hline 0.9 & 0.9 & 0.5 & 0.1 & 0.616414 & 51 \\
\hline 0.3 & 0.3 & 0.5 & 0.05 & 0.649967 & 32 \\
\hline 0.3 & 0.6 & 0.5 & 0.05 & 0.686454 & 40 \\
\hline 0.3 & 0.9 & 0.5 & 0.05 & 0.676481 & 41 \\
\hline 0.6 & 0.3 & 0.5 & 0.05 & 0.627259 & 45 \\
\hline 0.6 & 0.6 & 0.5 & 0.05 & 0.649168 & 48 \\
\hline 0.6 & 0.9 & 0.5 & 0.05 & 0.615139 & 48 \\
\hline 0.9 & 0.3 & 0.5 & 0.05 & 0.614936 & 48 \\
\hline 0.9 & 0.6 & 0.5 & 0.05 & 0.636954 & 54 \\
\hline 0.9 & 0.9 & 0.5 & 0.05 & 0.599240 & 54 \\
\hline 0.3 & 0.3 & 0.5 & 0.1 & 0.518484 & 23 \\
\hline 0.3 & 0.6 & 0.5 & 0.1 & 0.592122 & 32 \\
\hline 0.3 & 0.9 & 0.5 & 0.1 & 0.564593 & 32 \\
\hline 0.6 & 0.3 & 0.99 & 0.1 & 0.527925 & 24 \\
\hline 0.6 & 0.6 & 0.99 & 0.1 & 0.589566 & 32 \\
\hline 0.6 & 0.9 & 0.99 & 0.1 & 0.564456 & 33 \\
\hline 0.9 & 0.3 & 0.99 & 0.1 & 0.539383 & 24 \\
\hline 0.9 & 0.6 & 0.99 & 0.1 & 0.582916 & 32 \\
\hline 0.9 & 0.9 & 0.99 & 0.1 & 0.557999 & 34 \\
\hline 0.3 & 0.3 & 0.99 & 0.05 & 0.567959 & 28 \\
\hline 0.3 & 0.6 & 0.99 & 0.05 & 0.585832 & 33 \\
\hline 0.3 & 0.9 & 0.99 & 0.05 & 0.579936 & 34 \\
\hline 0.6 & 0.3 & 0.99 & 0.05 & 0.556921 & 28 \\
\hline 0.6 & 0.6 & 0.99 & 0.05 & 0.597425 & 33 \\
\hline 0.6 & 0.9 & 0.99 & 0.05 & 0.577321 & 36 \\
\hline 0.9 & 0.3 & 0.99 & 0.05 & 0.550236 & 28 \\
\hline 0.9 & 0.6 & 0.99 & 0.05 & 0.591618 & 35 \\
\hline 0.9 & 0.9 & 0.99 & 0.05 & 0.556443 & 37 \\
\hline
\end{tabular}

SECoS performs best over other methods such as probabilistic neural network (PNN) and support vector machine (SVM) in term of false negative as SECoS is able to learn and to adapt input pattern continuously. Even dough it true positive is less than SVM. SECoS is more robust in dealing with changes in error patterns Figure 7 [31].

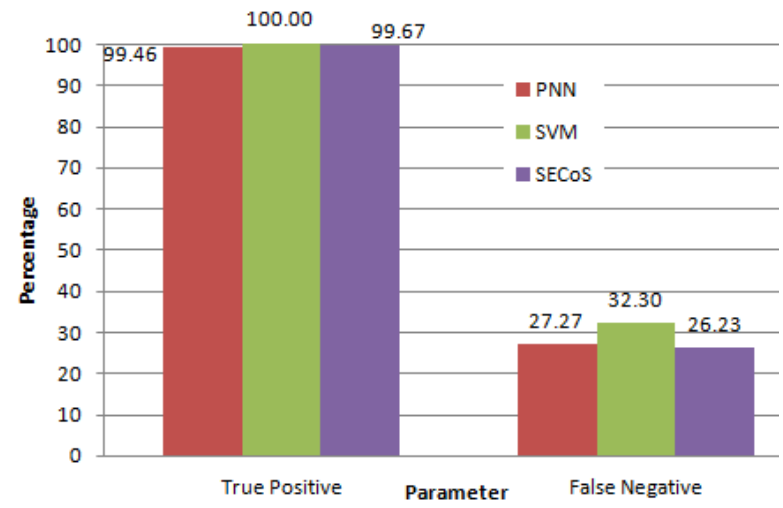

Figure 7. SECoS and other classifiers comparison 


\section{CONCLUSION}

In summary, this paper has discussed the classification of skin cancer with an image dataset in the form of malignant and benign skin images by using SECoS algorithm. In order to prepare the classifier input, grayscaling and HOG feature extraction were performed. The normalized Euclidean distance was employed as its performance is better than Hamming and Manhattan distances. The modified SECoS works well in performing continuous learning of the received and it changes hidden node adaptively. The evaluation process of the skin cancer disease was carried out on 136 datasets with 100 training and 36 testing data. Combination process of parameters in the classification obtained the smallest error in the learning rate 1 of 0.3 , learning rate 2 of 0.3 , when the sensitivity threshold is 0.5 and the error threshold is 0.1 . The minimum obtained MAPE is 0.5184845 and the number of hidden nodes is 23 nodes. Even dough true positive of SEcoS is smaller than SVM; its false negative is much better which proves that SECoS is more robust in handling frequent input pattern variations.

\section{ACKNOWLEDGEMENTS}

We would like to thank to the University of Muhammadiyah Sumatera Utara (UMSU) and Universitas Sumatera Utara (USU) for the support provided during this research.

\section{REFERENCES}

[1] S. S. Nikam, "A Comparative Study of Classification Techniques in Data Mining Algorithms," Int. Journal Mod. Trends Eng. Res., vol. 4, no. 7, pp. 58-63, 2017, doi: 10.21884/ijmter.2017.4211.vxayk.

[2] E. M. Hassib, A. I. El-Desouky, L. M. Labib, and E.-S. M. El-kenawy, "WOA + BRNN: An imbalanced big data classification framework using Whale optimization and deep neural network," Soft Comput., v. 24, no. 8, pp. 55735592, 2020, doi: 10.1007/s00500-019-03901-y.

[3] A. Lubis, M. Lubis, and A.- Khowarizmi, "Optimization of distance formula in K-Nearest Neighbor method," Bulletin. Elec. Eng. Informatics, vol. 9, no. 1, pp. 326-338, 2020, doi: 10.11591/eei.v9i1.1464.

[4] S. Prayudani, A. Hizriadi, Y. Lase, Y. Fatmi, and A. Khowarizmi, "Analysis Accuracy of Forecasting Measurement Technique on Random K-Nearest Neighbor using MAPE and MSE,” J. Physic Conf. Ser., vol. 1361, no. 1, pp. 0-8, 2019, doi: 10.1088/1742-6596/1361/1/012089.

[5] V. Lopez-vazquez, J. Lopez-guede, S. Marini, E. Fanelli, E. Johnsen, and J. Aguzzi, "Video image enhancement and machine learning pipeline for underwater animal detection and class. at cabled observatories," Sensors, vol. 20, no. 3, 2020, doi: 10.3390/s20030726.

[6] A. P. Gopi, R. Jyothi, V. L. Narayana, and K. Sandeep, "Classification of tweets data based on polarity using improved RBF kernel of SVM,” In. Jour. Info. Technol., 2020, doi: 10.1007/s41870-019-00409-4.

[7] A. E. Maxwell, T. A. Warner, and F. Fang, "Implementation of machine learning classification in remote sensing: An applied review," Int. J. of Remote Sensing, vol. 39, no. 9, pp. 2784-2817, 2018, doi: 10.1080/01431161.2018.1433343

[8] American Cancer Society, Cancer Facts \& Figures 2020, "Special Section: Cancer in Adolescents and Young Adults," 2020, [Online serial]. Available: http:// www.cancer.org. [Accessed Sept. 2, 2020].

[9] M. E. Celebi, N. Codella, and A. Halpern, "Dermoscopy image analysis: overview and future directions," IEEE jour. of biomed. and health informatics, vol. 23, no. 2, pp. 474-478, 2019, doi: 10.1109/JBHI.2019.2895803.

[10] M. N. Munjal and S. Bhatia, "A Novel Technique for Effective Image Gallery Search using Content Based Image Retrieval System," 2019 Int. Conf. on Machine Learning, Big Data, Cloud and Parallel Computing, Faridabad, India, pp. 25-29, 2019, doi: 10.1109/COMITCon.2019.8862206.

[11] Y. M. Chung, C. S. Hu, A. Lawson, and C. Smyth, "Topological approaches to skin disease image analysis". In 2018 IEEE Int. Conf. on Big Data (Big Data), IEEE, pp. 100-105, 2008, doi: 10.1109/BigData.2018.8622175

[12] A. Khowarizmi, I. Nasution, M. Lubis, and A. Lubis, "The effect of a SECoS in crude palm oil forecasting to improve business intelligence," Bull. of El. Eng. and Informatics, vol. 9, no. 4, pp. 1604-1611, 2020, doi: 10.11591/eei.v9i4.2388

[13] M. J. Watts, "Fuzzy Rule Extraction From Simple Evolving Connectionist Systems," Int. Journal on Comput. Intell. Appl., vol. 04, no. 03, pp. 299-308, 2004, doi: 10.1142/s146902680400132x.

[14] S. Silva, P. Costa, M. Gouvea, A. Lacerda, F. Alves, and D. Leite, "High impedance fault detection in power distr. systems using wavelet transform and evolving neural network," Electr. Pow. Syst. Res., vol. 154, pp. 474-483, 2018, doi: 10.1016/j.epsr.2017.08.039.

[15] N. K. Kasabov, "Evolving connectionist systems for adaptive learning and knowledge discovery: Trends and directions," Knowledge-B. Syst., v. 80, pp. 24-33, 2015, doi: 10.1016/j.knosys.2014.12.032.

[16] M. J. Watts, "Evolving Connectionist Systems can predict outbreaks of the aphid Rhopalosiphum padi," Int. Conf. Neural Networks, pp. 646-650, 2014, doi: 10.1109/IJCNN.2014.6889755.

[17] M. J. Watts, "Sleep learning and Max-Min aggregation of Evolving Connectionist Systems," Proc. Int. Jt. Conf. Neural Networks, vol. 2016-Octob, pp. 4325-4330, 2016, doi: 10.1109/IJCNN.2016.7727764.

[18] N. Kasabov, "Evolving connectionist systems: The knowledge engineering approach," Evol. Connect. Syst. Kn. Eng. Approach (Second Ed.), pp. 1-457, 2007, doi: 10.1007/978-1-84628-347-5. 
[19] S. Agrawal and J. Agrawal, "Survey on anomaly detection using data mining techniques," Procedia Comp.. Sci., vol. 60, no. 1, pp. 708-713, 2015, doi: 10.1016/j.procs.2015.08.220.

[20] A. Khowarizmi, "Modified of Simple Evolving Connectionist System using Distance Formulas," Universitas Sumatera Utara, 2017.

[21] A. Khowarizmi, A. M. Lubis, and A. R. Lubis, "Classification of Tajweed Al-Qur'an on Images Applied Varying Normalized Distance Formulas,” no. 3, pp. 21-25, 2020, doi: 10.1145/3396730.3396739.

[22] I. Mierswa, K. Morik, "Automatic feature extraction for classifying audio data", Machine learning, Vol. 58, No.2, p.127-149, doi: 10.1007/s10994-005-5824-7.

[23] G. Forman and E. Kirshenbaum, "Extremely fast text feature extraction for classification and indexing," Int. Conf. Inf. Knowl. Manag. Proc., pp. 1221-1230, 2008, doi: 10.1145/1458082.1458243.

[24] L. Benuskova, N. Kasabov, L. Benuskova, and N. Kasabov, "Evolving Connectionist Systems (ECOS)," Springer, 2007, doi: 10.1007/978-0-387-48355-9_5.

[25] A. Sebbar, S. Heddam, O. Kisi, L. Djemili, and L. Houichi, "Comparison of Evolving Connectionist Systems (ECoS) and Neural Networks for Modelling Daily Pan Evaporation from Algerian Dam Reservoirs," Heidelberg: Springer Berlin Heidel., pp. 1-19, doi: 10.1007/698_2020_527.

[26] A. Khowarizmi, I. R. Nasution, M. Lubis, and A. R. Lubis, "The effect of a secos in crude palm oil forecasting to improve business intelligence," Bull. Electr. Eng. Informatics, vol. 9, no. 4, pp. 1604-1611, 2020, doi: 10.11591/eei.v9i4.2388.

[27] N. K. Kasabov, L. Goh, and M. Sullivan, "Integrated Prognostic Profiles: Combining Clinical and Gene Expression Information through Evolving Connectionist Approach,” in Inf. Proc. and Liv. Systems, vol. 2, pp. 695-707, 2005, doi: 10.1142/9781860946882_0010.

[28] S. Kalouche, "Vision-Based Classification of Skin Cancer using Deep Learning," Stanford, 2016.

[29] R. F. Rahmat, A. Rizki, A. F. Alharthi, and R. Budiarto, "Big data forecasting using evolving multi-layer perceptron," 2016 4th Saudi Int. C. Inf. Technol. (Big Data Anal. KACSTIT) 2016, 2016, doi: 10.1109/KACSTIT.2016.7756069.

[30] A. Khowarizmi, O. S. Sitompul, S. Suherman, and E. B. Nababan, "Measuring the Accuracy of Simple Evolving Connectionist System with Varying Distance Formulas," in J. of Phy.: Conference Series, 2017, vol. 930, no. 1, doi: 10.1088/1742-6596/930/1/012004.

[31] S. Silva, "High impedance fault detection in power distribution systems using wavelet transform and evolving neural network." Elec. Pow. Syst. Research, vol. 154, pp. 474-483, 2018, doi: 10.1016/j.epsr.2017.08.039.

\section{BIOGRAPHIES OF AUTHORS}

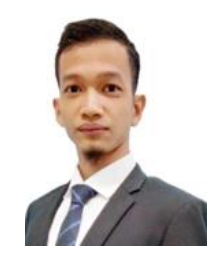

Al-Khowarizmi is the head of Information Technology Department within CSIT Faculty in Universitas Muhammadiyah Sumatera Utara (UMSU), Medan Indonesia. He obtained his MSc in Information Technology from University of Sumatera Utara in 2017. His research interest lies on artificial intelligence and machine learning, data science and business applications.

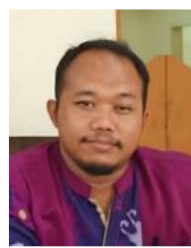

Suherman is the head of master degree program in Electrical Engineering within Universitas Sumatera Utara. He obtained his B.Eng, MSc and PhD from Universitas Sumatera UtaraIndonesia, RMIT-Australia and De Montfort University-UK subsequently in 2000, 2009 and 2013. He joined some courses in Melbourne University, Ilmenau University of Technology and European Bioinformatics Institute from 2008 to 2012. His research interests are in applied communication system, renewable energy, and computer science. 